\title{
Dissociable effects of irrelevant context on 2D and 3D grasping
}

\author{
Aviad Ozana $^{1}$ - Tzvi Ganel ${ }^{1}$
}

Published online: 3 November 2017

(C) The Psychonomic Society, Inc. 2017

\begin{abstract}
Grasping movements directed toward real objects are typically unaffected by irrelevant aspects of the object and its surroundings, indicating that such interactions are based on analytic processing of object shape and size. However, recent findings show that grasping directed toward two-dimensional (2D) objects is subjected to perceptually mediated effects of relative shape and size. It is unclear however, whether contextdependent processing - a hallmark of visual perception - affects $2 \mathrm{D}$ grasping in the same fashion. Here, we explored this possibility by comparing the influence of a newly discovered contextual effect on 2D and on 3D grasping. According to the range of standard effect (RSE), the perceptual resolution for a stimulus depends on the range of the other stimuli presented within the same session, with higher resolution obtained under narrow compared to wide context range. In two experiments, participants were asked to grasp 3D and 2D objects embedded in a wide or a narrow range. The results showed that, unlike 3D grasping, which was immune to contextual information, the resolution during $2 \mathrm{D}$ grasping was significantly modulated by the range of the irrelevant context. The findings suggest that visuomotor control directed to 2D objects is intruded by irrelevant perceptual information, making it contextdependent
\end{abstract}

Keywords Effect of stimulus range - Perception and action . Two-dimensional objects $\cdot$ Object perception $\cdot$ Contextual processing

Tzvi Ganel

tganel@bgu.ac.il

1 Department of Psychology, Ben-Gurion University of the Negev, 8410500 Beer-Sheva, Israel
The proposal that visuomotor control toward objects is mediated by a distinct functional and neuroanatomical visual system has received ample empirical support (Goodale \& Milner, 1992; Ungerleider \& Mishkin, 1982; Whitwell, Milner, \& Goodale, 2014). Behavioral data indicate that guided actions do not follow the same psychophysical principles that govern people's visual perception (Goodale \& Ganel, 2015). In particular, perceptual judgments are typically subjected to relative and holistic processing style of objects' size and shape (Garner \& Felfoldy, 1970; Hochstein \& Ahissar, 2002; Navon, 1977), and are affected by task-irrelevant contextual information (Gregory, 1970). Yet, grasping movements directed to real objects are typically performed in an analytic and selective fashion, immune to the effects of relative and contextual information (Aglioti, DeSouza, \& Goodale, 1995; Ganel, Chajut, \& Algom, 2008; Ganel \& Goodale, 2003, but see Kopiske, Bruno, Hesse, Schenk, \& Franz, 2016).

Recent technological developments have led to a growing interest in detailing the representations that mediate the visual processing of virtual, 2D objects. Several studies that compared visuomotor control toward 2D and 3D objects suggest that the functional distinction that characterizes vision-foraction and vision-for-perception does not generalize to visuomotor control directed to 2D objects. Unlike the grasping of real objects, grasping movements directed at 2D objects are bound to holistic processing style of object shape, and are affected by relative information about object size (Freud \& Ganel, 2015; Holmes \& Heath, 2013; Hosang, Chan, Davarpanah Jazi, \& Heath, 2016; Ozana \& Ganel, 2017). For example, Weber's law, a fundamental principle of visual perception, states that the resolution to visual size is relative, and decreases in a linear fashion with size. Yet, unlike as for perception, the kinematics of the fingers during 3D grasping do not obey to Weber's law (Ganel, 2015; Ganel et al., 2008; Ganel, Freud \& Meiran, 2014). The kinematics during 2D 
grasping were, however, shown to adhere to Weber's law (Holmes \& Heath, 2013; Hosang et al., 2016; Ozana \& Ganel, 2017), which indicates that, for 2D grasping, visual information is processed in a relative rather than in an analytic (absolute) manner.

Based on these findings, it has been suggested that, unlike as for actions directed to real objects, actions directed to $2 \mathrm{D}$ objects are intruded by irrelevant perceptual information about relative size and shape (Freud \& Ganel, 2015; Ozana \& Ganel, 2017). It is still unclear, however, whether $2 \mathrm{D}$ grasping can be characterized as strictly perceptual and relative in nature along all aspects of visual processing (Freud \& Ganel, 2015). Specifically, it is unclear whether contextual processing - a hallmark of relative perception-extends to grasping movements directed toward 2D objects. Previous studies that looked at the effects of illusory context on 2D grasping have generally shown illusions had little or no effects on 2D grasping (Stöttinger, Soder, Pfusterschmied, Wagner, \& Perner, 2010; Vishton, Rea, Cutting, \& Nunez, 1999, for other studies that examined the effect of context on 2D pointing, see Krigolson \& Heath, 2004; Krigolson, Clark, Heath, \& Binsted, 2007). Yet, these studies did not directly compare 2D and 3D grasping, which makes it difficult to draw firm conclusion as to the relationship between the effects of context and the dimensionality of the visual stimulus. To best of our knowledge, only one previous study has examined this issue and directly compared the effects of contextual illusory information on 2D and 3D grasping (Kwok \& Braddick, 2003). In Kwok and Braddick's study, context was manipulated using the size-contrast Ebbinghaus illusion, in which a target circular object is embedded in the context of small or large circular flankers (Aglioti et al., 1995). The findings showed that similarly to $3 \mathrm{D}$ grasping, trajectories for 2D objects were immune to the contextual effects of the Ebbinghaus illusion. However, accumulating evidence casts doubts on whether or not the Ebbinghaus illusion can be used as an effective tool to tap potential dissociations between perception and action (for recent examples, see Kopiske et al., 2016; Whitwell \& Goodale, 2016). Therefore, the question of whether 2D grasping is affected by contextual information calls for further investigation. The purpose of the current study was to test this issue by focusing at the resolving power of the response-a basic psychophysical aspect of performance (Ganel et al., 2008; Namdar, Ganel, \& Algom, 2016; Zitron-Emanuel \& Ganel, 2017). To this purpose, we tested the effect of contextual information on the visual resolution of 2D (and 3D) grasping using a simple psychophysical tool, recently developed in our laboratory (Namdar, Algom, \& Ganel, 2017; Namdar et al., 2016).

Recently, Namdar et al., (2016) reported the discovery of a new contextual effect on visual resolution. According to the range of standards effect (RSE), visual resolution for a target stimulus depends on the range of the contextual stimuli presented within the same session (for a similar idea in the domain of stimulus identification, see Ward, Armstrong, \& Golestani, 1996). In particular, the difference threshold, or just noticeable difference (JND) for a given stimulus is affected by the range of other standards tested for resolution within the same experimental session. Participants were presented with a $40 \mathrm{~mm}$ length target object, when other standards tested within the same session were either wide $(20,60 \mathrm{~mm})$ or narrow $(35,45 \mathrm{~mm})$ in range. The results showed that stimulus resolution was affected by context range. When the stimulus was presented in a wide context range, resolution went in the same direction of the context range and JNDs were larger compared to the narrow-range condition (Namdar et al., 2016). These results were replicated and extended in a follow-up study that compared the effect of stimulus range between perception and action (Namdar et al., 2017). Now, participants were asked to either grasp the objects or to perform perceptual estimations of their length. JNDs for action and perception were derived using the psychophysical method of adjustment (Baird \& Noma, 1978; Ganel et al., 2008; Gescheider, 1985). For perception, JNDs for the common standard were again larger in the wide range condition, and hence affected by the range of the other stimuli tested for resolution. However, for grasping, JNDs for the common standard were not affected by stimulus range, indicating that unlike for perception, the resolution during (3D) grasping is immune to the effects of contextual information (Namdar et al., 2017; Goodale \& Ganel, 2015).

The goal of the current study was to examine whether the RSE, a contextual effect of human resolving power in perception (but not in action), extends to visually guided actions directed to $2 \mathrm{D}$ objects. To this end, JNDs during 2D grasping were compared under conditions in which the other, contextual 2D targets were either close (narrow range) or distant (wide range) in size. In Experiment 1, we measured the RSE during grasping movements performed toward 2D line drawings of rectangular objects. Our goal in Experiment 2 was to replicate and extend the results of Experiment 1 for grasping movements toward realistic photos of the objects. Would 2D grasping, in a similar manner to $3 \mathrm{D}$ grasping, be immune to the effect of contextual stimulus range?

\section{Experiment 1}

\section{Methods}

\section{Participants}

A group of 22 right-handed students (seven males) with normal or corrected to normal vision participated in the experiment. The average age of the participants was 24 years $(\mathrm{SD}=$ 2.1 years). They all provided informed consent to participate in the experiment and received the equivalent of US \$7 for 
their participation. Ethics were approved by the Ben-Gurion University (BGU) Psychology Ethics Committee.

\section{Apparatus and stimuli}

Participants sat in front of a black tabletop on which a monitor was placed horizontally on the table surface (Fig. 1). Computercontrolled PLATO goggles (Translucent Technologies, Toronto, ON) with liquid-crystal shutter lenses were used to control stimulus exposure time. Grip scaling was recorded by an Optotrak Certus device (Northern Digital, Waterloo, ON). The apparatus tracked the 3D position of three active infra-red light emitting diodes attached separately to the participant's index finger, thumb and wrist (200 $\mathrm{Hz}$ sampling rate).

The target objects were 2D filled line drawings of rectangular objects. Objects were constant in width $(5 \mathrm{~mm})$ and varied along their height $(20 \mathrm{~mm}, 35 \mathrm{~mm}, 40 \mathrm{~mm}, 45 \mathrm{~mm}$ or $60 \mathrm{~mm}$ ). The stimuli were presented at the center of the $1366 \times$ 768 resolution LCD display (Samsung, $60 \mathrm{~Hz}$ refresh rate) against a black background (see Fig. 1). Stimuli were presented at a $30 \mathrm{~cm}$ distance from the movement starting point. Trial sequence and stimuli presentation were controlled with SuperLab software (version 5.0.4, Cedrus Corporation, San Pedro, CA).

\section{Procedure}

The procedure was similar to the one used in a previous study in which we looked at the effect of context range on grasping trajectories (Namdar et al., 2017). Prior to each trial, participants rested their index finger and thumb pinched together on a start button, while the goggles were set to the translucent state. Participants were instructed to touch the upper and lower edges of the target object. They were told to initiate their movement upon hearing a "go" tone. They were then asked to keep their fingers still at the end point for an additional $1 \mathrm{~s}$ prior to returning to the

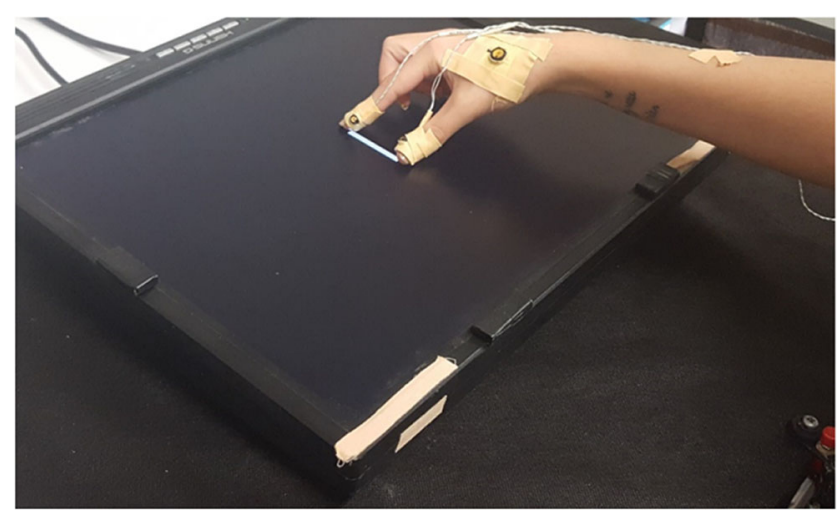

Fig. 1 Experimental setup used in Experiment 1. Participants were asked to perform grasping movements toward a set of rectangular twodimensional (2D) objects presented on a computer screen while their fingers position was tracked starting position. Each trial began with an opening of the goggles, which remained open for $3000 \mathrm{~ms}$; allowing full visual feedback during the trial. The "go" tone was presented $1000 \mathrm{~ms}$ after the initial opening of the goggles.

Following a few practice trials and equipment-calibration, each participant performed the experiment under the narrow range and the wide range conditions, which were presented in two separate blocks. The narrow-range block included stimuli of $35 \mathrm{~mm}, 40 \mathrm{~mm}$, and $45 \mathrm{~mm}$ in length. The wide-range block task included stimuli of $20 \mathrm{~mm}, 40 \mathrm{~mm}$, and $60 \mathrm{~mm}$ in length. The $40 \mathrm{~mm}$-length stimulus, which was presented in both blocks, served as the common standard upon which the effect of context has been employed. Each block contained 60 pseudo-random consecutive experimental trials (20 repetitions of each object, a total of 120 trials). Block order was counterbalanced across participants.

\section{Data analysis and design}

On each trial, we recorded the 3D trajectories of the fingers. The recorded data was analyzed offline using MATLAB software (Version 9.0, The Mathworks, Natick, MA). Movement onset was set at the point in time at which the index finger velocity exceeded $100 \mathrm{~mm} / \mathrm{s}$. Movement offset was set at the point in time at which the fingers' velocity fall below $50 \mathrm{~mm} / \mathrm{s}$. To analyze the movement trajectory, we divided each trial's trajectory into 11 intervals equal in length $(0-100 \%)$ and calculated the average aperture in each interval point. The withinsubject standard deviations of the aperture during the point in which maximum grip apertures (MGAs) were achieved were calculated separately for each object and were used as the measure for JNDs (Ganel et al., 2008). MGAs were determined only if they were achieved at the second part $(50-100 \%)$ of the normalized movement. This criterion was set in order to avoid a possible concern related to the MGA data in $2 \mathrm{~d}$ grasping. Specifically, the second part of the trajectory has been shown to be more stable and more representative in terms of the sensitivity of the aperture to object's size (both in 2D and 3D grasping, see Holmes \& Heath, 2013), and in terms of smaller fluctuations in aperture velocity (which could increase statistical noise, see Foster \& Franz, 2013; Ganel, 2015). In most cases, this issue is not problematic because the MGA is (normally) achieved at a late stage of the movement (Jakobson \& Goodale, 1991; Jeannerod, 1984). However, during 2D grasping, we noticed several instances in which the MGA was achieved early in the trajectory, for example, cases of "double peaks" in aperture in which the first, early peak was larger than the second one. Such cases could lead to averaging MGAs across completely different segments of the movement, which could increase statistical noise and prevent 
effective comparison between the MGA data in 2D and $3 \mathrm{D}$ grasping. We note that, regardless of the way the MGA data is analyzed, the general trend of results as well as the significance of the RSE were always maintained.

For the main analysis, the effect of context range (two levels) on the target $(40 \mathrm{~mm})$ stimulus served as the withinsubject independent variable. In an additional analysis that focused at the possible adherence of 2D grasping to Weber's law, Object size (three levels) and Context range (two levels) served as within-subject independent variables (for a similar analysis, see Namdar et al., 2017). The average aperture and the JND at the point in time in which MGAs were achieved served as the dependent variables. To extend the analysis to other aspects of the movements beyond MGAs, we also analyzed the average aperture trajectories for the different objects throughout the movement. In addition, different aspects of movement times (MT) were also analyzed. Response time (RT) was the average time to initiate the movement from the presentation of the "go" tone. Time to MGA (TMG) and overall MT were also calculated.

A correction for outliers was applied on the data by eliminating trials in which the MGAs were 2.5 standard deviations (SD) higher or lower than average for each object size. This correction resulted in the exclusion of less than $2 \%$ of the trials from the final analysis.

\section{Results}

$J N D s$

JNDs during the point in time in which MGAs were achieved for each of the target objects are presented in Fig. 2. As can be

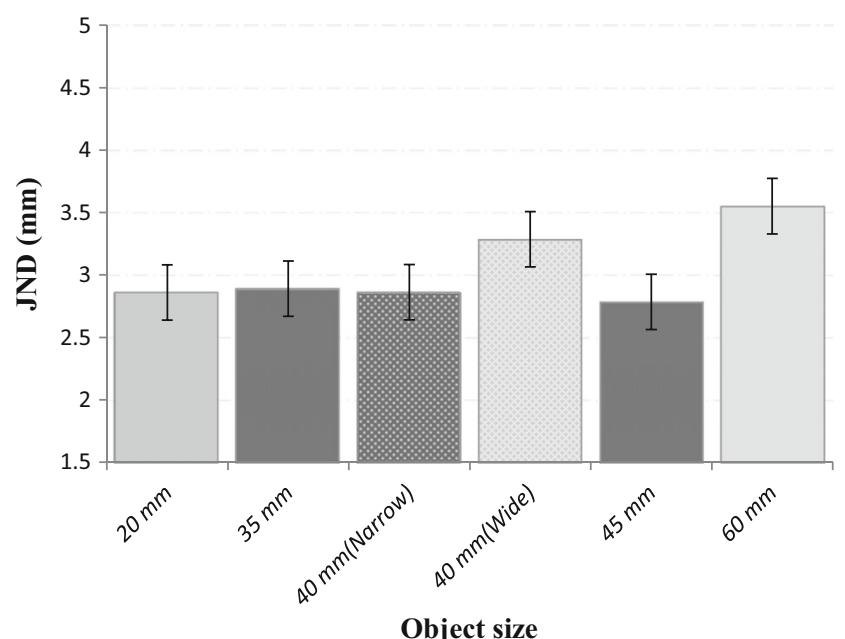

Fig. 2 Just noticeable differences (JNDs) in Experiment 1. JNDs for the common standard were affected by context, indicating an increase in resolution in the narrow range compared to the wide context. Error bars Confidence intervals in repeated measures ANOVAs (Jarmasz \& Hollands, 2009) seen in the figure, the JND for the common standard was modulated by stimulus range, and was larger in the wide compared to the narrow-range condition $[\mathrm{t}(21)=2.17, P<.05)$. These findings show that similarly to perceptual estimations, JNDs during 2D grasping are modulated by the context in which the target stimulus is embedded.

In an additional analysis, we examined the adherence to Weber's law within the two context ranges. A repeated measures ANOVA showed a main effect of context range $\left[F_{(1,21)}\right.$ $\left.=5, P<.05, \eta_{\mathrm{p}}{ }^{2}=0.19\right]$. The main effect for object size was not significant $\left[\mathrm{F}_{(2,42)}=1.8, P>.1\right]$. The interaction between range and size was significant $\left[\mathrm{F}_{(1.5,32.9)}=5.2, P<.05, \eta_{\mathrm{p}}{ }^{2}=\right.$ $0.20]$. Planned comparisons of the linear component of size revealed that this interaction resulted from different relationships between JNDs and size. Specifically, JNDs linearly increased with size in the wide range condition $\left[\mathrm{F}_{(1,21)}=5, P<\right.$ $\left..05, \eta_{\mathrm{p}}{ }^{2}=0.19\right]$ conforming adherence to Weber's law, but not in the narrow range condition $\left[\mathrm{F}_{(1,21)}=0.7, p>.1\right]$. A similar pattern of results was obtained in a recent study from our laboratory in which we examined the adherence of perceptual manual estimations to Weber's law under the two context ranges (Namdar et al., 2017). Adherence to Weber's law was found in the wide but not in the narrow context range, presumably due to the relatively small differences in sizes between the objects in this condition, which did not allow sufficient sensitivity for detecting potential differences in perceptual resolution.

\section{Grip apertures}

Grip aperture during the point in time in which MGAs were achieved for each of the target objects, and movement trajectories of two representative subjects are presented in Fig. 3. As can be seen in the figure, grip apertures were sensitive to object size. A repeated measures ANOVA with Greenhouse-Geisser correction was conducted on the average grip aperture MGA data of all subjects, and revealed a main effect for context range $\left[\mathrm{F}_{(1,21)}=7.8, P<.05, \eta_{\mathrm{p}}{ }^{2}=\right.$ $0.27]$, and object size $\left[\mathrm{F}_{(1.2,26.2)}=721, P<.05, \eta_{\mathrm{p}}{ }^{2}=0.97\right]$. In addition, an interaction between context range and object size showed that the slope relating MGAs and object size in the narrow range condition was different than the slope in the wide range condition $\left[\mathrm{F}_{(1.2,26.3)}=706, P<.05, \eta_{\mathrm{p}}{ }^{2}=\right.$ 0.97]. Planned comparisons showed that hand aperture during MGAs increased with object size in the narrow range (42 $\mathrm{mm}, 45 \mathrm{~mm}$ and $48 \mathrm{~mm}$ for the small, medium, and big object, respectively) $\left[\mathrm{F}_{(1,21)}=238, P<.05, \eta_{\mathrm{p}}{ }^{2}=0.91\right]$, and in the wide range condition $(34 \mathrm{~mm}, 46 \mathrm{~mm}, 60 \mathrm{~mm})\left[\mathrm{F}_{(1,21)}\right.$ $\left.=907, P<.05, \eta_{\mathrm{p}}{ }^{2}=0.97\right]$, conforming sensitivity to size. A paired samples $t$-test was conducted to compare the average aperture for the common standard (40 mm stimulus) in the narrow range and wide range conditions. Average aperture in the narrow range did not statistically differ from that 


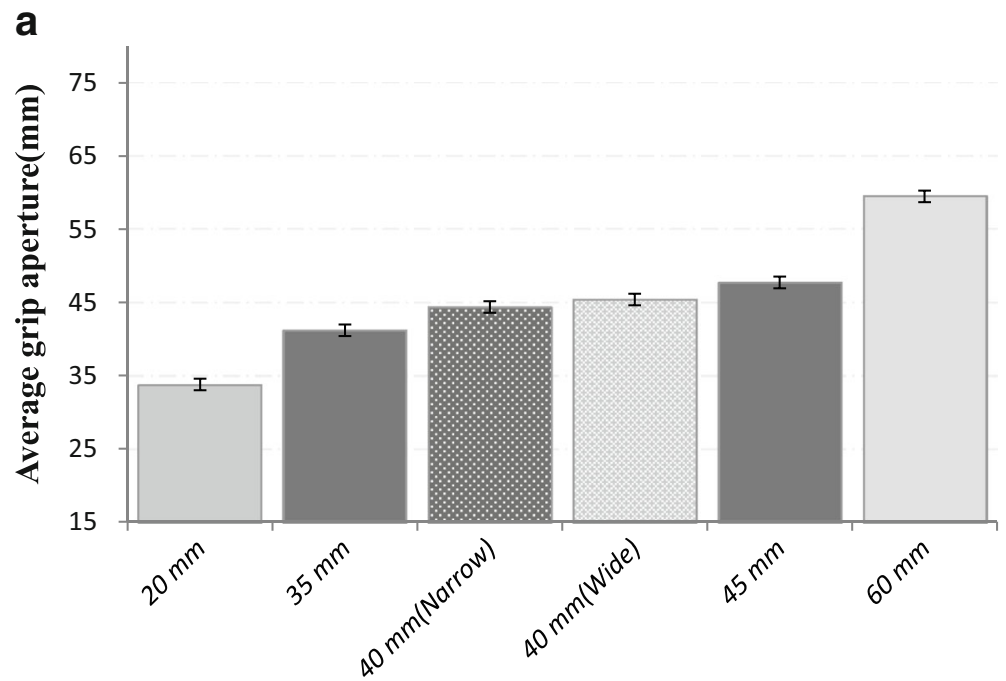

Object size
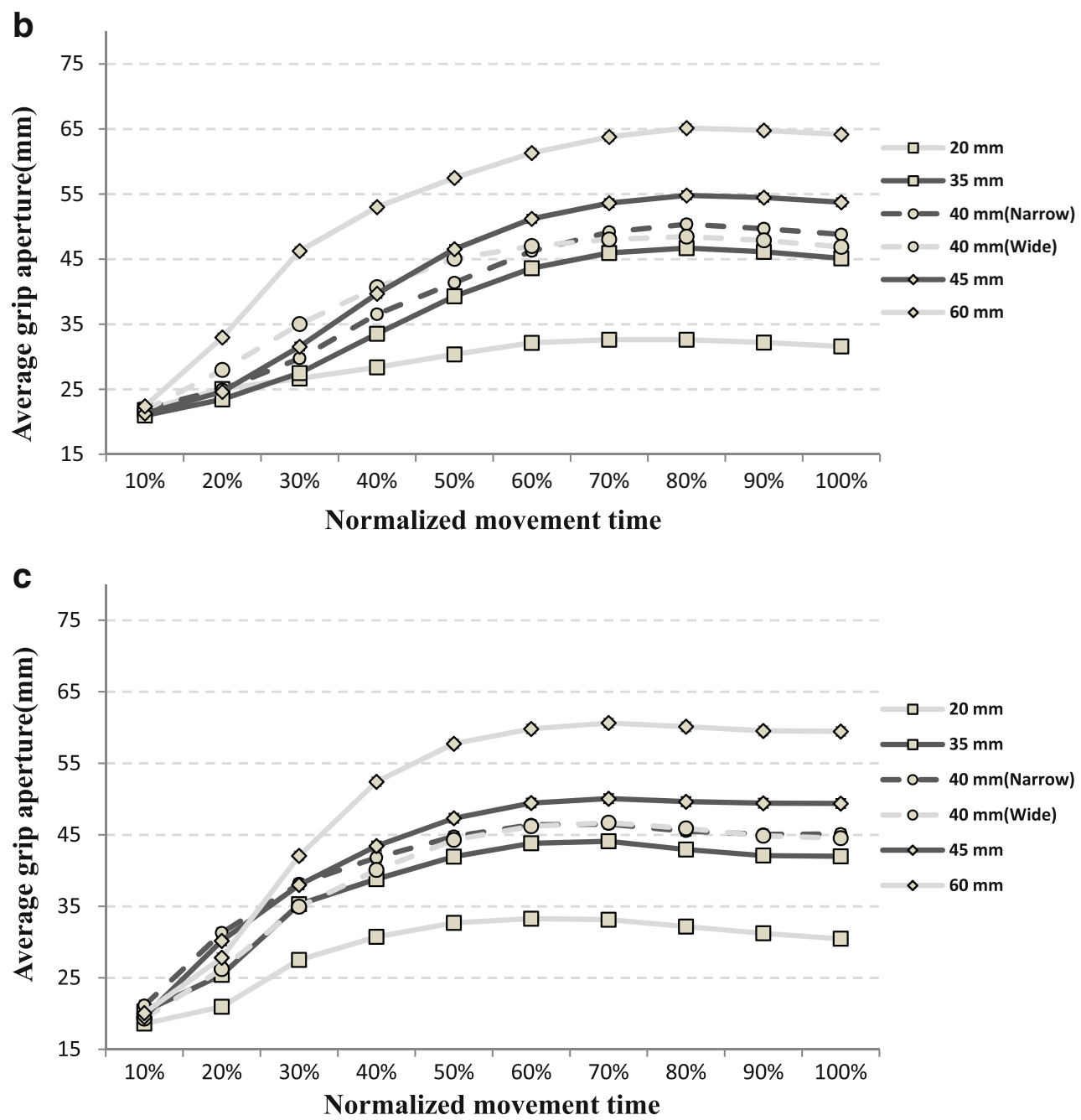

Fig. 3 a Average grip apertures at the point of maximum grip apertures (MGA). b, c Example of normalized movement trajectories of two representative participants. Grip apertures were sensitive to object size. Error bars Confidence intervals in repeated measures ANOVAs (Jarmasz \& Hollands, 2009)

of the wide range $[45 \mathrm{~mm}, 46 \mathrm{~mm}$, respectively, $\mathrm{t}(21)=$ $0.64, P>.1]$. Mean response times including RTs, times to
MGA (TMGAs), and MTs for each of the target objects are presented in Table 1. 
Table 1 Mean response time (RT), time to maximum grip aperture (TMGA), and movement time (MT) $[ \pm 1$ standard deviation (SD)] for each of the objects in Experiment 1

\begin{tabular}{llllll}
\hline & $20 \mathrm{~mm}$ & $35 \mathrm{~mm}$ & $40 \mathrm{~mm}$ (narrow) & $40 \mathrm{~mm}$ (wide) & $45 \mathrm{~mm}$ \\
\hline RT & $451 \pm 188$ & $434 \pm 148$ & $453 \pm 168$ & $452 \pm 181$ & $434 \pm 139$ \\
TMGA & $477 \pm 136$ & $626 \pm 126$ & $660 \pm 109$ & $645 \pm 162$ & $713 \pm 144$ \\
MT & $768 \pm 139$ & $880 \pm 122$ & $899 \pm 110$ & $915 \pm 161$ & $778 \pm 148$ \\
\hline
\end{tabular}

The results of Experiment 1 suggest that 2D grasping is subjected to effects of context. In a similar way to perceptual estimations (Namdar et al., 2016, 2017), resolution was modulated by the range of the other stimuli presented in the block. This pattern of results suggests that the visuomotor system, which in other cases was found to be immune to task-irrelevant contextual information, is intruded by this information when 2D objects are presented for grasp. It can be argued therefore, that similarly to other perceptual attributes that affect 2D grasping but not affect 3D grasping (Freud \& Ganel 2015; Holmes \& Heath, 2013), context has similar effects via perceptual processing that intrudes into action.

Before embracing the idea that $2 \mathrm{D}$ grasping is modulated by contextual information, a potential concern needs to be addressed. Namely, the stimuli used in Experiment 1 were simple line drawings of objects, which lack the rich texture and explicit reference to depth that are naturally embedded in real objects. It can be therefore argued that irrelevant visual aspects of the scene, rather than differences inherent to the nature of the task, contributed to the susceptibility of grasping to irrelevant effects of context (for a similar discussion related to the adherence to Weber's law, see Ozana \& Ganel, 2017). Experiment 2 was designed to replicate and extend the results of Experiment 1 by using 2D stimuli that were matched in terms of visual detail and complexity to the real 3D objects presented within the same experiment. This allowed us to include an additional control condition in which real 3D objects were presented and to directly compare the effect of context range on $2 \mathrm{D}$ and $3 \mathrm{D}$ grasping.

\section{Experiment 2}

\section{Participants}

Thirty right-handed students (ten males, average age: 23.5 years:, $\mathrm{SD}=1.5$ ) participated in the experiment for the equivalent of US $\$ 10$ or course credit. The results of one participant were removed from the analysis because he failed to follow the experimental instructions. All participants provided informed consent for their participation in the experiment and ethics were approved by the BGU psychology ethic committee.

\section{Stimuli}

The 3D objects were five plastics rods identical in lengths and width to the objects presented in Experiment $1(5 \mathrm{~mm}$ in depth). The objects were placed on the center surface of the monitor, against a black background. The 2D objects were high resolution photos of the $3 \mathrm{D}$ objects. The objects were displayed on the monitor against a black background. The photos were photographed from the participant's point of view using an 8-megapixel camera. They were later cropped and modified using Adobe Photoshop to be similar in terms of appearance and dimensions to the 3D objects.

\section{Procedure and design}

The procedure was similar to that used in Experiment 1 with a few exceptions. First, a 3D grasping condition was included, in which participants were asked to touch the upper and lower edges of the target object without lifting it up. Second, high resolution photos of the objects were presented in the $2 \mathrm{D}$ condition. Lastly, to equate, as much as possible, response times between 2D and 3D grasping, a secondary auditory cue was sound, $1250 \mathrm{~ms}$ following the presentation of the "go" tone. Participants were instructed to complete their movement prior to the presentation of the second tone. Our goal was to motivate the participants to perform their movements within similar time frames for $2 \mathrm{D}$ and $3 \mathrm{D}$ grasping, without interfering with their natural prehension.

As in Experiment 1, each block contained 60 experimental trials, resulting in a total of 240 trials for the four experimental blocks. The fingers average aperture and JND at the point in time in which MGAs were achieved served as the dependent variables. Less than $2 \%$ of the trials were defined as outliers and excluded from the analysis. Object type (2D $\backslash 3 \mathrm{D})$ and context range (Narrow $\backslash$ Wide) served as the independent, within-subject variables in the main analysis. For the secondary analysis that looked at adherence to Weber's law, object size was also included as a withinsubject variable. 


\section{Results}

\section{Just noticeable differences}

JNDs for the different-sized objects during 2D and 3D grasping are presented in Fig. 4. As can be seen, the results of the 2D grasping condition closely replicated the results of Experiment 1. In particular, the JNDs of the common standard were again larger in the wide compared to the narrow context, in line with the RSE. In sharp contrast, JNDs during 3D grasping were not affected by context range or by object size and were similar across the two experimental conditions.

To test the effects of context on 2D and 3D grasping, a repeated measures ANOVA was conducted on the JND data for the common standard, with context range and object type as independent variables. The analysis revealed a main effect of object type $\left[\mathrm{F}_{(1,28)}=18.9, P<\right.$

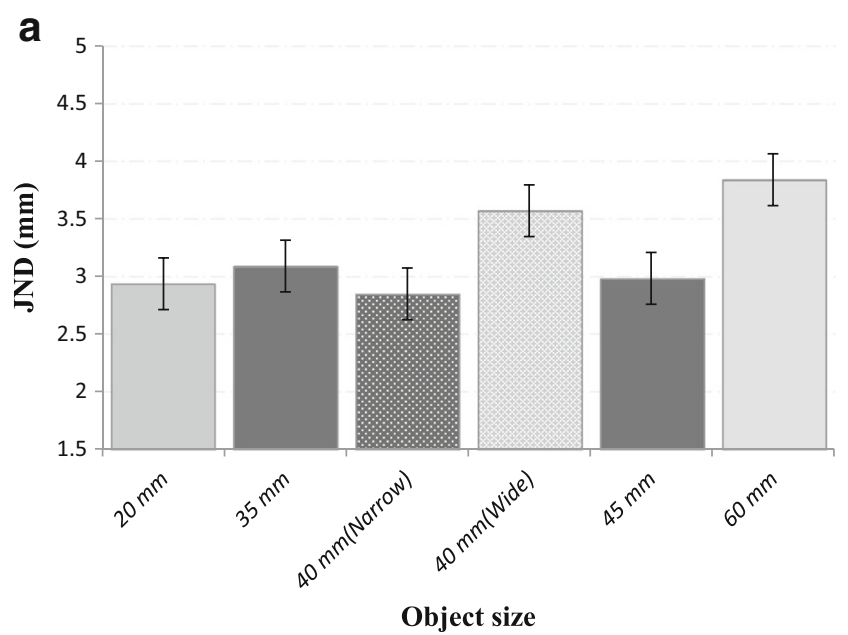

b

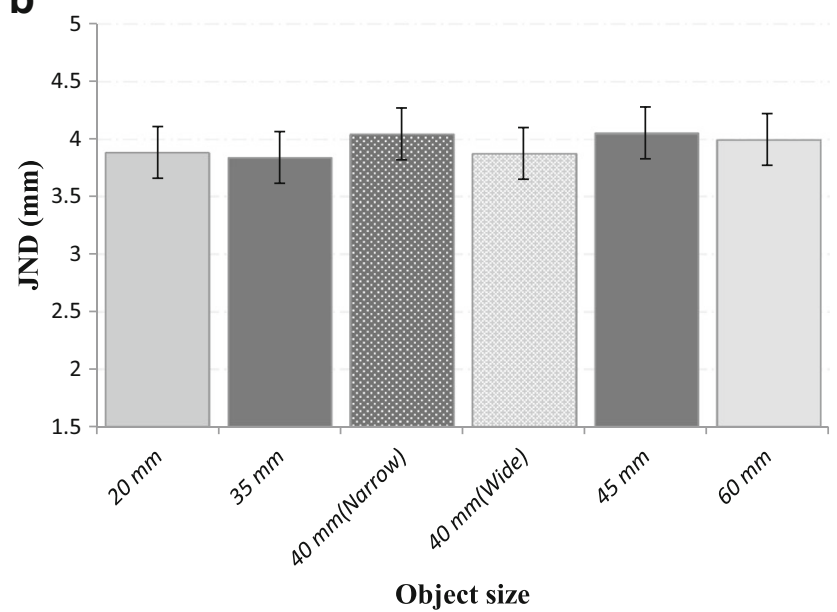

Fig. 4 JNDs in Experiment 2 in $\mathbf{a} 2 \mathrm{D}$ and $\mathbf{b}$ three-dimensional (3D) grasping. During 2D grasping, JNDs were again modulated by context range and were larger in the wide range compared to the narrow range condition. Unlike in 2D grasping, JNDs during 3D grasping were not affected by context range. Error bars Confidence intervals in repeated measures ANOVAs (Jarmasz \& Hollands, 2009) $\left..05 \eta_{\mathrm{p}}{ }^{2}=0.40\right]$, with overall larger JNDs during 3D grasping. The main effect of range was not significant $\left[F_{(1,28)}=\right.$ 2.5, $P>$.1]. More importantly, a significant interaction between object type and context range confirmed that range had different effects in $2 \mathrm{D}$ and $3 \mathrm{D}$ grasping $\left[\mathrm{F}_{(1,28)}=5.8, P<.05 \eta_{\mathrm{p}}{ }^{2}=0.17\right]$. A paired samples $t$-test revealed that range affected the stimulus resolution in the $2 \mathrm{D}$ condition, with larger JNDs in the wide compared to the narrow context $[2.8 \mathrm{~mm}, 3.5 \mathrm{~mm}$, respectively, $\mathrm{t}(28)=$ 2.77, $P<.05$ ]. Unlike for 2D grasping, 3D grasping was not affected by context range $(\mathrm{t}(28)=0.68, P>.1)$, replicating previous results (Namdar et al., 2017).

To test the adherence of JNDs to Weber's law, an additional analysis was performed on the JND data of the different-sized objects in 2D and in 3D grasping. A repeated measures ANOVA revealed a significant main effect of object type $\left[\mathrm{F}_{(1,28)}=25, \mathrm{p}<.05, \eta_{\mathrm{p}}{ }^{2}=0.47\right]$ and object size $\left[\mathrm{F}_{(2,56)}=\right.$ $\left.4.1, P<.05, \eta_{\mathrm{p}}{ }^{2}=0.12\right]$. The main effect of context range was not significant $\left[\mathrm{F}_{(1,28)}=2.2, P>.1\right]$, as well as the interaction between object type and object size $\left[\mathrm{F}_{(2,56)}=0.7, P>.1\right]$. A marginal interaction was found between context range and object size $\left[\mathrm{F}_{(2,56)}=3, P<.1, \eta_{\mathrm{p}}{ }^{2}=0.09\right]$. A significant interaction was found between object type and context range $\left[\mathrm{F}_{(1,28)}=6.5, P<.05, \eta_{\mathrm{p}}{ }^{2}=0.17\right]$, indicating that range had different effects in 2D and in 3D grasping. Finally, a 3-way interaction was found between object type, context range, and object size $\left[\mathrm{F}_{(2,56)}=3.6, P<.05, \eta_{\mathrm{p}}{ }^{2}=0.11\right]$. To further test the adherence of the trajectories to Weber's law for each object type, two separate repeated measures ANOVAs were conducted on the JND data for 2D and for 3D grasping, with object size and context range as independent variables. For 2D grasping, a significant main effect was found for context range $\left[\mathrm{F}_{(1,28)}=6.4, P<.05, \eta_{\mathrm{p}}{ }^{2}=0.18\right]$, and object size $\left[\mathrm{F}_{(2,56)}=\right.$ $\left.3.2, P<.05, \eta_{\mathrm{p}}{ }^{2}=0.10\right]$. In addition, similar to the results of Experiment 1, a significant interaction was found between object size and range $\left[\mathrm{F}_{(2,56)}=7.1, P<.05, \eta_{\mathrm{p}}{ }^{2}=0.20\right]$. As in Experiment 1, planned comparisons of the linear component of size showed a linear increase with size in the wide range condition $\left[\mathrm{F}_{(1,28)}=9.6, P<.05\right.$, $]$ but not in the narrow range condition $\left[\mathrm{F}_{(1,28)}=0.4, P>.1\right]$. For $3 \mathrm{D}$ objects, the main effects of context range $\left[\mathrm{F}_{(1,28)}=0.1, P>.1\right]$, object size $\left[\mathrm{F}_{(2,56)}=0.9, P>.1\right]$ and the interaction $\left[\mathrm{F}_{(2,56)}=0.2, P>\right.$ .1] were not significant.

\section{Grip apertures}

Grip aperture at the point in time in which MGAs were achieved for all objects during 2D and 3D grasping are presented in Fig. 5. As can be seen in the figure, apertures were sensitive to object size. A repeated measures ANOVA with Greenhouse-Geisser correction of the grip aperture revealed main effects of object type $\left[\mathrm{F}_{(1,28)}=\right.$ $\left.202, P<.05, \eta_{\mathrm{p}}{ }^{2}=0.87\right]$, context range $\left[\mathrm{F}_{(1,28)}=15, P\right.$ 


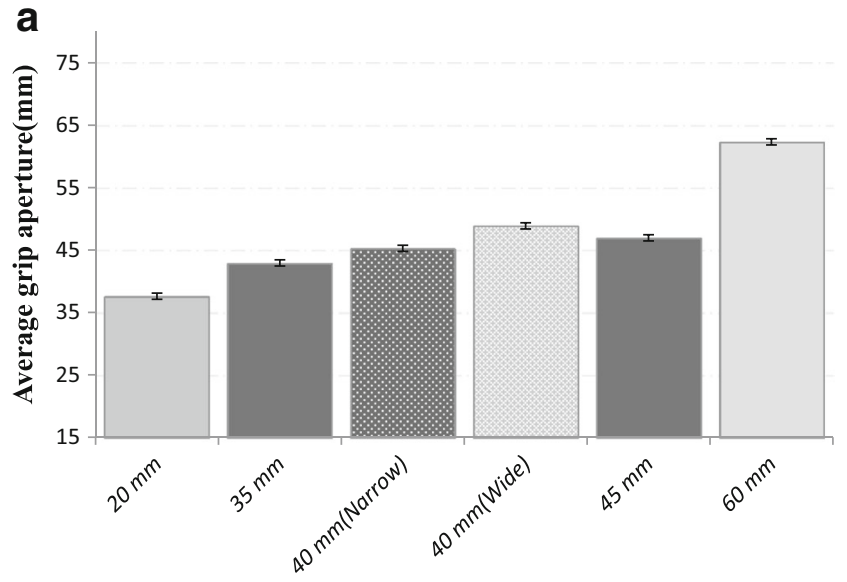

b

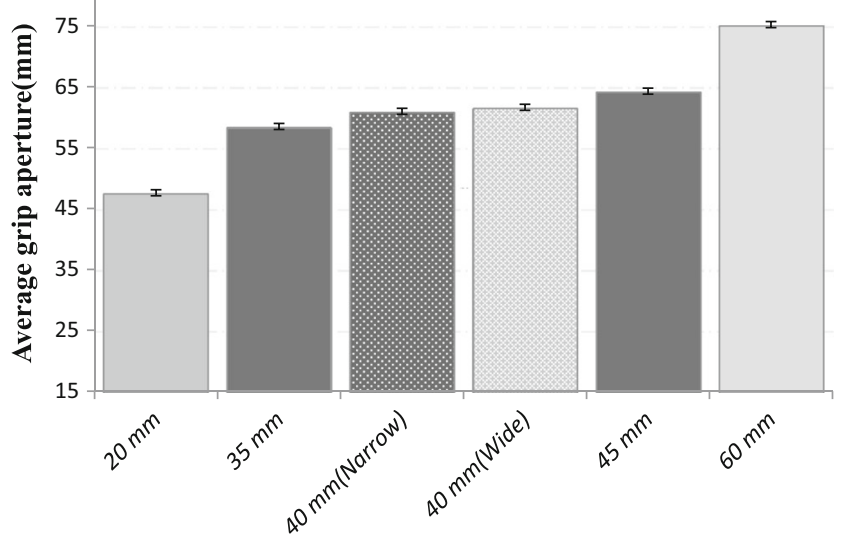

Object size

Fig. 5 Average grip aperture in Experiment 2 in $\mathbf{a} 2 \mathrm{D}$ and $\mathbf{b}$ 3D grasping. Grip apertures were sensitive to object size. Error bars Confidence intervals in repeated measures ANOVAs (Jarmasz \& Hollands, 2009)

$\left.<.05, \eta_{\mathrm{p}}{ }^{2}=0.35\right]$, and object size $\left[\mathrm{F}_{(1.3,38.4)}=1375, P<\right.$ $\left..05, \eta_{\mathrm{p}}{ }^{2}=0.98\right]$, as well as significant interactions between type and range $\left[\mathrm{F}_{(1,28)}=12.6, P<.05, \eta_{\mathrm{p}}{ }^{2}=\right.$ $0.31]$ type and size $\left[\mathrm{F}_{(1.4,39.3)}=16, P<.05, \eta_{\mathrm{p}}{ }^{2}=0.35\right]$ and size and range $\left[\mathrm{F}_{(1.3,37.3)}=718, P<.05, \eta_{\mathrm{p}}{ }^{2}=0.96\right]$. The 3-way interaction between range, size, and type was also significant $\left[\mathrm{F}_{(2,56)}=5.8, P<.05, \eta_{\mathrm{p}}{ }^{2}=0.17\right]$. Mean response times including RTs, TMGAs, and MTs for each of the target objects are presented in Table 2 .
The results of Experiment 2 replicate and extend those of Experiment 1. For 2D grasping, the findings again show compliance to the RSE, which has been now generalized to a situation in which high resolution photos are presented as targets. In contrast to $2 \mathrm{D}$ grasping, and in agreement with previous results (Namdar et al., 2017), grasping trajectories toward real, 3D objects were immune to contextual effects. Taken together with the findings of Experiment 1, the results provide the first demonstration that unlike as in $3 \mathrm{D}$ grasping, visual resolution during $2 \mathrm{D}$ grasping is modulated by context.

\section{General discussion}

The aim of the present study was to examine if grasping movements toward 2D objects can be resistant to irrelevant effects of context. Our findings show otherwise; unlike as in $3 \mathrm{D}$ grasping, visual resolution during $2 \mathrm{D}$ grasping was significantly affected by contextual information. In particular, the visual resolution during $2 \mathrm{D}$ grasping was influenced by the stimulus range in which the target stimulus was embedded. JNDs for the common standard were lower in the narrow range, in agreement with the RSE. This effect was not limited to simple 2D line drawings of objects and has been generalized to situations in which grasping was directed to highresolution object photos (Experiment 2, for a similar result related to the adherence to Weber's law, see Ozana \& Ganel, 2017). The findings indicate that the compliance to the RSE is a general property of 2D visuomotor control.

Namdar et al. (2017) recently showed that visually guided actions directed at 3D objects are not influenced by the RSE. Unlike for perceptual judgments, JNDs for the common standard were unaffected by the context range in which it was embedded, indicating the operation of a selective processing style, typical to visuomotor control toward real objects (Goodale \& Ganel, 2015). The results of the current study provide further evidence for the idea that such selective, analytic processing style does not characterize 2D grasping (Freud \& Ganel, 2015; Holmes \& Heath, 2013). The resolving power to detect changes along the size of a common 2D target was modulated by contextual information. Therefore, our

Table 2 Mean RT, TMGA, and MT ( $\pm 1 \mathrm{SD})$ for each of the objects in Experiment 2

\begin{tabular}{|c|c|c|c|c|c|c|c|}
\hline & & $20 \mathrm{~mm}$ & $35 \mathrm{~mm}$ & $40 \mathrm{~mm}$ (narrow) & $40 \mathrm{~mm}$ (wide) & $45 \mathrm{~mm}$ & $60 \mathrm{~mm}$ \\
\hline \multirow[t]{3}{*}{$2 \mathrm{D}$ grasping } & RT & $435 \pm 109$ & $469 \pm 114$ & $464 \pm 110$ & $441 \pm 75$ & $469 \pm 115$ & $444 \pm 93$ \\
\hline & TMGA & $596 \pm 96$ & $640 \pm 100$ & $662 \pm 134$ & $694 \pm 165$ & $684 \pm 109$ & $779 \pm 109$ \\
\hline & MT & $1015 \pm 163$ & $969 \pm 159$ & $970 \pm 155$ & $1022 \pm 165$ & $980 \pm 150$ & $1045 \pm 185$ \\
\hline \multirow[t]{3}{*}{ 3D grasping } & $\mathrm{RT}$ & $440 \pm 91$ & $424 \pm 70$ & $430 \pm 70$ & $448 \pm 85$ & $440 \pm 75$ & $447 \pm 95$ \\
\hline & TMGA & $553 \pm 121$ & $577 \pm 110$ & $591 \pm 107$ & $589 \pm 123$ & $609 \pm 145$ & $643 \pm 135$ \\
\hline & MT & $934 \pm 130$ & $964 \pm 124$ & $969 \pm 123$ & $945 \pm 117$ & $966 \pm 126$ & $970 \pm 133$ \\
\hline
\end{tabular}


findings suggest that visuomotor control of $2 \mathrm{D}$ objects is governed by a relative processing style and affected by context. In addition, the current findings converge to those of a recent study (Namdar et al., 2017) to suggest that the RSE can be used as reliable measure for probing the representations that mediate visual perception and action.

These results are consistent with the results of previous studies that showed that $2 \mathrm{D}$ grasping is subjected to the effects of relative processing of size (Holmes \& Heath, 2013; Hosang et al., 2016) and shape (Freud \& Ganel, 2015). Freud and Ganel used the Garner speeded-classification task to show that unlike as for 3D grasping (Ganel \& Goodale, 2003, Ganel \& Goodale, 2014, Schum, Franz, Jovanovic, \& Schwarzer, 2012; but see Eloka, Feuerhake, Janczyk, \& Franz, 2015; Hesse \& Schenk, 2013), 2D grasping relies on holistic shape representation in which one dimension of an object cannot be processed independently from other dimensions belonging to the same object. The current results extend these findings and provide additional support for the idea that actions toward virtual, 2D stimuli rely, at least at part, on contextual processing. It can be argued therefore, that various aspects of visual perception that do not intrude into actions when real, 3D objects are presented for grasp, have a significant effect on grasping movements directed at 2D objects.

Unlike the findings of the current work, Kwok and Braddick (2003) reported that 2D grasping is not biased by contextual effects inherent to the Ebbinghaus illusion. Similarly to 3D grasping, fingers aperture was adjusted to the real size of the targets, regardless of the sizes of surrounding flankers. Yet, recent findings questioned the validity of Ebbinghaus illusion as a tool to probe the nature of the visual processes that mediate a motor response (Kopiske et al., 2016; but see Whitwell \& Goodale, 2016). When different visual illusions were used in 2D grasping, the findings were less consistent, showing either small effects of the illusions in some cases (the horizontal-vertical illusion, see Vishton et al., 1999), or no effects of the illusions in other cases (the diagonal illusion, see Stöttinger et al., 2010; Stöttinger, Aigner, Hanstein, \& Perner, 2009). We again note, however, that previous studies (with the exception of Kwok and Braddick, 2003), did not compare the effects of the illusions on $2 \mathrm{D}$ and $3 \mathrm{D}$ grasping within the same experimental design, which does not allow drawing a firm conclusion as to the effects of stimulus dimensionality on the successability of grasping movements to (illusory) context. Further research in which the effects of different visual illusions would be direclty compared for $2 \mathrm{D}$ and for $3 \mathrm{D}$ grasping is required in order to explore this issue.

Unlike in studies of visual illusions, which focus on the bias of the average response, the current study focused on response resolution, measured by the variblity of the withinsubject response (Ganel et al., 2008; Namdar et al., 2016, 2017). In the context of the discussion on the adherence of grasping movements to Weber's law, it has been proposed that several factors should be considered when looking at the varbility of the grip aperture. One such factor is the possiblity of biomechnical constraints during grasping (Utz, Hesse, Aschenneller, \& Schenk, 2015). Such constraints could lead to ceiling effects when looking at the varaiblity of the responses for consderably large obejcts, beyond the limit of one's reach (Bruno, Uccelli, Viviani, \& De'Sperati, 2016; Heath, Manzone, Khan, \& Jazi, 2017). Researches should be conscious, therefore to possible ceiling effects when designing experiments that tap visual resolution during grasping (Ganel, Namdar, \& Mirsky, 2017). We note, however, that biomechanical constraints could not have affected the results of the current study because the target objects were similar in size (40 $\mathrm{mm}$ ) across the two ranges. Furthermore, the size of the common standard was within the range of comfort grasps and does not allow a potential threat of biomechanical constrains (see Heath et al., 2017). We also note that the results of the current study as well as of previous stuides that showed significant adhernece of 2D grasping to Weber's law are in odds with the suggestion that adherence to Weber's law cannot be generally found during motor control due to an inherent noise (Löwenkamp, Gärtner, Haus, \& Franz, 2015).

The results of the current study are also of relevance for understanding the visoumortor abilites of a neuropsychological patient suffering from visual-form agnosic. In particular, patient DF, who suffers from severe impairments in her perceptual processing and from difficulties performing perceptual estimations of size, has intact ability to compute object size during 3D grasping (Goodale, Milner, Jakobson, \& Carey, 1991). Interestingly, however, DF's normal sensitivity to size during 3D grasping extends to situations in which line drawings of 2D objects are presented as targets (Westwood, Danckert, Servos, \& Goodale, 2002). Although these findings seem to be at odds with the findings of the current study, and with other behavioral evidence of differences between 2D and 3D grasping (Freud \& Ganel, 2015; Holmes \& Heath, 2013; Ozana \& Ganel, 2017), it is important to note that that perceptual processing in visual form agnosia may be different from that of neurologically intact individuals. The fact that perceptual processing is severly impaired in Patient DF could account for why relative and contextual perceptual information does not affect her 2D grasping performance. Therefore, given that grasping directed to $2 \mathrm{D}$ objects can be considered as a visuomotor task that is intruded by irrelevant perceptual processing (Ozana \& Ganel, 2017), it is possible that DF's relatively intact visuomotor abilities allow her to perform the task normally (compared to controls) in simple grasping tasks that do not involve irrelevant perceptual processing.

The findings of the current study also converge with previous reports to suggest that grasping movements toward stimuli in the real-world and grasping movements toward $2 \mathrm{D}$ objects are functionally (and neuroanatomically) distinct. 
Unlike as in real-object grasping, visually guided actions toward 2D targets were shown to be subjected to various effects of relative and holistic processing inherent to visual perception (Freud \& Ganel, 2015; Holmes \& Heath, 2013; Hosang et al., 2016; Ozana \& Ganel, 2017). Moreover, recent fMRI findings indicate that the mere presentation of real objects and objects' photos evoke different patterns of activations (Snow et al., 2011; Snow, Strother, \& Humphreys, 2014). Recently, Freud and his colleagues (Freud et al., 2017) have extended these ideas to the domain of grasping and used fMRI to show that 2D and 3D grasping are modulated by differential patterns of neural activation. Indeed, Freud and his colleagues (2017) propose that the neural mechanisms that mediate real grasping could be dissociated, at least in part, from those that mediate $2 \mathrm{D}$ grasping, which is in line with the behavioral dissociation reported in the present study.

Here, as well as in previous papers that looked at the properties of visuomotor control toward 2D targets, grasping was used as a model task to test for potential differences between the nature of processing of $2 \mathrm{D}$ and $3 \mathrm{D}$ objects. It is relevant to note, however, that although grasping has been shown to be informative for comparing 2D and 3D visuomotor control, grasping movements are typically applied when 3D targets are presented as targets (for discussion, see Freud \& Ganel, 2015). Indeed, the task of 2D grasping could be ambiguous in terms of its specific demands, and could be differently interpreted by different people, which could also lead to a potential variability in the pattern of movement trajectory. Some previous studies chose to approach this potential issue of task ambiguity by instructing participants to simply "grasp" the 2D objects, just as in 3D grasping (Freud \& Ganel, 2015; Holmes \& Heath, 2013). This approach has the advantage of using similar instructions across the 2D and 3D grasping tasks as well as of allowing for individual differences in 2D (and 3D) grasping to be expressed. However, it is also disadvantageous in that it could lead to unwarranted effects of variability, especially during $2 \mathrm{D}$ grasping. In the present study as well as in a recent study from our laboratory (Ozana \& Ganel, 2017), we chose to apply a different approach. In particular, we specifically asked the participants to touch the edges of the 2D target objects at the end of each grasping movement (similar instructions were given in the $3 \mathrm{D}$ grasping task). The advantage of this design is that it uses a clear set of instructions during $2 \mathrm{D}$ and $3 \mathrm{D}$ grasping and helps preventing possible effects of noise due to individual differences in movement variability. Yet, it is important to note that regardless of the specific task instructions used in 2D grasping, previous studies have consistently reported that unlike as in 3D grasping, 2D grasping is affected by perceptual, relative processing style (Freud \& Ganel, 2015; Holmes \& Heath, 2013; Ozana \& Ganel, 2017). Although it would be interesting for future research to test the specific effect of instructions on 2D grasping, current empirical evidence strongly suggests that regardless of the specific task used, 2D grasping is performed in relative, contextual processing style.

It can be argued that asking participants to place their fingers on the edges of objects during 2D grasping may encourage them to use a "double pointing" strategy during grasping (Smeets \& Brenner, 1999). According to Smeets and Brenner's account on visuomotor control, grasping is achieved via double pointing, which is predicted not to involve the computation of object size, but merely that of the discrete location of the fingers. Such location-based computations are not expected to be modulated by perceptual effects related to size or magnitude, including the RSE and including adherence to Weber's law (Smeets \& Brenner, 2008). We note, however, that the present findings, that show that $2 \mathrm{D}$ (but not 3D) grasping movements are modulated by the two effects of (irrelevant) size (see also Ozana \& Ganel, 2017) cannot be accounted for by a simple account of double pointing.

Another potential difference between 2D and 3D grasping, which has been suggested to account for the different processing types observed in both tasks, is the availability of haptic feedback. At the end of a successful 3D grasping movement, the participant receives haptic feedback from the edges of the target object, which provide informative cues about its size and material. Such cues, however, are absent during 2D grasping, for which the participant receives only partial, general cues about object size upon touching the flat surface of the computer screen (Ozana \& Ganel, 2017). It has been suggested that the availability of tactile cues allows the visuomotor system to operate in its selective mode and to evade the influence of Weber's law (Hosang et al., 2016). In particular, Hosang and her colleagues (2016) suggested that, when valid tactile feedback is provided following the termination of a grasping trial, grasping movements toward 2D objects can be performed analytically, violating Weber's law. We note, however, that recent findings from our laboratory have separated between different types of tactile cues which could have differential effects on grasping performance toward real objects. In particular, we showed that when tactile cues are not provided at the end of the movement, and grasping is performed in thin air, the trajectories adhere to Weber's law, which is in line with Hosang et al.'s (2016) conclusions. However, the results also showed that when real objects (but not 2D objects) are presented for view, general tactile feedback (received from a transparent glass surface which was placed on top of the target object) is sufficient to allow analytic processing during grasp (Ozana \& Ganel, 2017). Given that the nature of the tactile feedback provided in Ozana and Ganel's study (in 3D grasping) resembles the feedback provided during $2 \mathrm{D}$ grasping, it can be argued that the nature of the tactile feedback alone does not determine grasp selectivity. In other words, it is more likely that interactions between the dimensionality of the target object (e.g., 2D or 3D) and the 
nature of available tactile cues (e.g., full haptic feedback, general tactile feedback, or no feedback at all), affect the susceptibility of grasping movements to irrelevant relative information.

To summarize, the current results provide evidence that unlike as in $3 \mathrm{D}$ grasping, grasping movements directed to 2D objects is susceptible to the effect of irrelevant contextual information. Together with growing evidence from behavior and neuroimaging studies, the current findings propose that the processes that mediate $2 \mathrm{D}$ visuomotor control can be fundamentally distinct than those that mediate visuomotor control directed at real objects. The findings also propose that when people direct their actions toward 2D, virtual objects, their trajectories may be less precise due to their increased susceptibility to the effects of irrelevant perceptual information that disrupts normal visuomotor control during grasping.

Acknowledgments We thank Shira Simon for her assistance in running the experiments. This study was supported by an Israel Science Foundation (ISF) grant 274/15 to Tzvi Ganel and to Daniel Algom.

\section{References}

Aglioti, S., DeSouza, J. F. X., \& Goodale, M. A. (1995). Size-contrast illusions deceive the eye but not the hand. Current Biology, 5(6), 679-685.

Baird, J. C., \& Noma, E. J. (1978). Fundamentals of scaling and psychophysics. New York: Wiley.

Bruno, N., Uccelli, S., Viviani, E., \& de'Sperati, C. (2016). Both visionfor-perception and vision-for-action follow Weber's law at small object sizes, but violate it at larger sizes. Neuropsychologia, 91, 327-334.

Eloka, O., Feuerhake, F., Janczyk, M., \& Franz, V. H. (2015). Garnerinterference in left-handed awkward grasping. Psychological Research, 79(4), 579-589.

Foster, R. M., \& Franz, V. H. (2013). Inferences about time course of Weber's Law violate statistical principles. Vision Research, 78, 56-60.

Freud, E., \& Ganel, T. (2015). Visual control of action directed toward two-dimensional objects relies on holistic processing of object shape. Psychonomic Bulletin \& Review, 22(5), 1377-1382.

Freud, E., Macdonald, S. N., Chen, J., Quinlan, D. J., Goodale, M. A., \& Culham, J. C. (2017). Getting a grip on reality: Grasping movements directed to real objects and images rely on dissociable neural representations. Cortex.

Ganel, T. (2015). Weber's law in grasping. Journal of Vision, 15(8), 18.

Ganel, T., Chajut, E., \& Algom, D. (2008). Visual coding for action violates fundamental psychophysical principles. Current Biology, 18(14), 599-601.

Ganel, T., Freud, E., \& Meiran, N. (2014). Action is immune to the effects of Weber's law throughout the entire grasping trajectory. Journal of Vision, 14(7). doi:https://doi.org/10.1167/14.7.11.doi

Ganel, T., \& Goodale, M. A. (2003). Visual control of action but not perception requires analytical processing of object shape. Nature, 426(6967), 664-667. doi:https://doi.org/10.1038/nature02156

Ganel, T., \& Goodale, M. A. (2014). Variability-based Garner interference for perceptual estimations but not for grasping. Experimental Brain Research, 232(6), 1751-1758.

Ganel, T., Namdar, G., \& Mirsky, A. (2017). Bimanual grasping does not adhere to Weber's law. Scientific Reports, 7, 6467.
Garner, W. R., \& Felfoldy, G. L. (1970). Integrality of stimulus dimensions in various types of information processing. Cognitive Psychology, 1(3), 225-241.

Gescheider, G. A. (1985). Psychophysics: Method, theory, and application, vol 12. Hillsdale: Erlbaum.

Goodale, M. A, \& Ganel, T. (2015). Different modes of visual organization for perception and for action. Oxford Handbook of Perceptual Organization, 3(1), 1-19. doi:https://doi.org/10.1093/oxfordhb/ 9780199686858.013.027

Goodale, M. A., \& Milner, A. D. (1992). Separate visual pathways for perception and action. Trends in Neurosciences, 15(1), 20-25.

Goodale, M. A., Milner, A. D., Jakobson, L. S., \& Carey, D. P. (1991). Perceiving the world and grasping it. A neurological dissociation. Nature, 349, 154-156.

Gregory, R. L. (1970). The intelligent eye. New York: McGraw-Hill.

Heath, M., Manzone, J., Khan, M., \& Jazi, S. D. (2017). Vision for action and perception elicit dissociable adherence to Weber's law across a range of 'graspable'target objects. Experimental Brain Research, (in press).

Hesse, C., \& Schenk, T. (2013). Findings from the Garner-paradigm do not support the "how" versus "what" distinction in the visual brain. Behavioural Brain Research, 239, 164-171.

Hochstein, S., \& Ahissar, M. (2002). View from the top: Hierarchies and reverse hierarchies in the visual system. Neuron, 36(5), 791-804. doi:https://doi.org/10.1016/S0896-6273(02)01091-7

Holmes, S. A., \& Heath, M. (2013). Goal-directed grasping: The dimensional properties of an object influence the nature of the visual information mediating aperture shaping. Brain and cognition, 82(1), 18-24. doi:https://doi.org/10.1016/j.bandc.2013.02.005

Hosang, S., Chan, J., Jazi, S. D., \& Heath, M. (2016). Grasping a 2D object: Terminal haptic feedback supports an absolute visuo-haptic calibration. Experimental Brain Research, 234(4), 945-954. doi: https://doi.org/10.1007/s00221-015-4521-4

Jakobson, L. S., \& Goodale, M. A. (1991). Factors affecting higher-order movement planning: A kinematic analysis of human prehension. Experimental Brain Research, 86(1), 199-208. doi:https://doi.org/ 10.1007/BF00231054

Jarmasz, J., \& Hollands, J. G. (2009). Confidence intervals in repeatedmeasures designs: The number of observations principle. Canadian Journal of Experimental Psychology/Revue canadienne de psychologie expérimentale, 63(2), 124.

Jeannerod, M. (1984). The timing of natural prehension movements. Journal of Motor Behavior, 16(3), 235-254. doi:https://doi.org/10. 1080/00222895.1984.10735319

Kopiske, K. K., Bruno, N., Hesse, C., Schenk, T., \& Franz, V. H. (2016). The functional subdivision of the visual brain: Is there a real illusion effect on action? A multi-lab replication study. Cortex, 79, 130-152. doi: https://doi.org/10.1016/j.cortex.2016.03.020

Krigolson, O., Clark, N., Heath, M., \& Binsted, G. (2007). The proximity of visual landmarks impacts reaching performance. Spatial Vision, 20(4), 317-336.

Krigolson, O., \& Heath, M. (2004). Background visual cues and memoryguided reaching. Human movement science, 23(6), 861-877.

Kwok, R. M., \& Braddick, O. J. (2003). When does the Titchener Circles illusion exert an effect on grasping?: Two-and three-dimensional targets. Neuropsychologia, 41(8), 932-940.

Löwenkamp, C., Gärtner, W., Haus, I. D., \& Franz, V. H. (2015). Semantic grasping escapes Weber's law. Neuropsychologia, 70, 235-245.

Namdar, G., Algom, D., \& Ganel, T. (2017). Dissociable effects of stimulus range on perception and action. Cortex. doi:https://doi.org/10. 1016/j.cortex.2016.12.017

Namdar, G., Ganel, T., \& Algom, D. (2016). The extreme relativity of perception: A new contextual effect modulates human resolving power. Journal of Experimental Psychology: General, 145(4), 509. 
Navon, D. (1977). Forest before trees: The precedence of global features in visual perception. Cognitive Psychology, 9(3), 353-383.

Ozana, A., \& Ganel, T. (2017). Weber's law in 2D and 3D grasping. Psychological Research, (in press).

Schum, N., Franz, V. H., Jovanovic, B., \& Schwarzer, G. (2012). Object processing in visual perception and action in children and adults. Journal of Experimental Child Psychology, 112(2), 161-177.

Smeets, J. B., \& Brenner, E. (1999). A new view on grasping. Motor control, 3(3), 237-271.

Smeets, J. B., \& Brenner, E. (2008). Grasping Weber's law. Current Biology, 18(23), R1089-R1090.

Snow, J. C., Pettypiece, C. E., McAdam, T. D., McLean, A. D., Stroman, P. W., Goodale, M. A., \& Culham, J. C. (2011). Bringing the real world into the fMRI scanner: Repetition effects for pictures versus real objects. Scientific Reports, 1, 130.

Snow, J. C., Strother, L., \& Humphreys, G. W. (2014). Haptic shape processing in visual cortex. Journal of Cognitive Neuroscience, 26(5), 1154-1167.

Stöttinger, E., Aigner, S., Hanstein, K., \& Perner, J. (2009). Grasping the diagonal: Controlling attention to illusory stimuli for action and perception. Consciousness and cognition, 18(1), 223-228.

Stöttinger, E., Soder, K., Pfusterschmied, J., Wagner, H., \& Perner, J. (2010). Division of labour within the visual system: Fact or fiction? Which kind of evidence is appropriate to clarify this debate? Experimental Brain Research, 202(1), 79-88.
Ungerleider, L. G., \& Mishkin, M. (1982). Two cortical visual systems. In D. J. Ingle, M. A. Goodale, \& R. J. W. Mansfield (Eds.), Analysis of visual behavior (pp. 549-586). Cambridge: MIT Press.

Utz, K. S., Hesse, C., Aschenneller, N., \& Schenk, T. (2015). Biomechanical factors may explain why grasping violates Weber's law. Vision Research, 111, 22-30.

Vishton, P. M., Rea, J. G., Cutting, J. E., \& Nuñez, L. N. (1999). Comparing effects of the horizontal-vertical illusion on grip scaling and judgment: Relative versus absolute, not perception versus action. Journal of Experimental Psychology: Human Perception and Performance, 25(6), 1659.

Ward, L. M., Armstrong, J., \& Golestani, N. (1996). Intensity resolution and subjective magnitude in psychophysical scaling. Attention, Perception, \& Psychophysics, 58(5), 793-801.

Westwood, D. A., Danckert, J., Servos, P., \& Goodale, M. A. (2002). Grasping two-dimensional images and three-dimensional objects in visual-form agnosia. Experimental Brain Research, 144(2), 262-267.

Whitwell, R. L., \& Goodale, M. A. (2016). Real and illusory issues in the illusion debate (Why two things are sometimes better than one): Commentary on Kopiske et al. (2016). Cortex, 88, 205-209.

Whitwell, R. L., Milner, A. D., \& Goodale, M. A. (2014). The two visual systems hypothesis: New challenges and insights from visual form agnosic Patient DF. Frontiers in Neurology, 5, 255.

Zitron-Emanuel, N., \& Ganel, T. (2017). The effect of food deprivation on human resolving power. Psychonomic Bulletin \& Review, (in press). 